\title{
Fenomenología de la memoria. Superando el antagonismo entre memoria individual y memoria colectiva*
}

\author{
Phenomenology of the memory. Overcoming the antagonism \\ between individual memory and collective memory
}

Fenomenologia da memória. Superando o antagonismo entre memória individual e memória coletiva

Fecha de entrega: 7 de octubre 2016

Fecha de evaluación: 9 de enero de 2017

Fecha de aceptación: 2 de febrero de 2017

Jochen Dreher ${ }^{* *}$

\section{Resumen}

El presente texto busca dar cuenta del sujeto en el cual opera la memoria, para ello se recurre a la memoria individual desde una perspectiva fenomenológica. Razón por la cual se tienen en cuenta consideraciones

* $\quad$ Este trabajo constituye un avance de los estudios que el profesor Dreher realiza en la Universidad de Konstanz en torno a la relación entre sociología y fenomenología, especialmente en el tema del poder y la subjetividad, tema que aqui es abordado desde la memoria en su consideración social e histórica. DOI:http://dx.doi.org/10.15332/s0120-8462.2017.0116.01

* Desde 2005 es director del Archivo de Ciencias Sociales de la Universidad de Konstanz (Alemania). Es docente de la Universidad de Konstanz y de la Universidad de St. Gallen. El presente artículo hace parte de los proyectos y temas de investigación que el autor desarrolla en campos como la sociología del conocimiento, la fenomenología y la sociología de la cultura, las organizaciones y la migración. Correo electrónico: Jochen.Dreher@uni-konstanz.de 
en torno a la memoria cultural, a la conciencia temporal, a la relevancia y a los sujetos del recurso. Así, en el texto se asume la validez para las ciencias sociales de la memoria externa con la cual es posible acceder al modo como las sociedades se perciben a sí mismas y se imaginan, teniendo en cuenta que la memoria individual es determinada por ese tratamiento colectivo del recuerdo. Con lo cual se muestra la diferencia entre las memorias de los individuos pero que en sus entrecruzamientos van construyendo una memoria general para cuyo puente se propone la teoría de la relevancia de Schütz.

Palabras clave: memoria, fenomenología, conciencia, relevancia, cultura.

\section{Abstract}

This paper seeks to inform of the subject in which memory operates, to do so individual memory is used from a phenomenological perspective. This is why considerations about cultural memory, the temporal awareness, the relevance and the subjects of the resource are taken into account. Thus, the text assumes the validity for the social sciences of the external memory with which it is possible to access the way in which societies perceive themselves and imagine themselves, taking into account that individual memory is determined by that collective treatment of the memory. This shows the difference between the memories of the individuals, but in their interlacing they construct a general memory for which bridge Schütz's theory of relevance proposed.

Keywords: memory, phenomenology, consciousness, relevance, culture. 


\section{Resumo}

O presente texto busca analisar ao sujeito no qual opera a memória, para isso se recorre à memória individual desde uma perspectiva fenomenológica. Razão pela qual se tem em conta considerações sobre a memória cultural, à consciência temporal, a relevância e aos sujeitos do recurso. Assim, no texto se assume a validez para as ciências sociais da memória externa pela qual é possível acessar ao modo como as sociedades se percebem a si mesmas e se imaginam, considerando que a memória individual é determinada por essa abordagem coletiva da lembrança. Pelo qual se apresenta a diferença entre as memórias dos indivíduos, mas que nos seus entrecruzamentos vão construindo uma memória geral para cuja ponte se propõe a teoria da relevância de Schütz.

Palavras-chave: memória, fenomenologia, consciência, relevância, cultura. 


\section{Planteamiento del problema}

Las reflexiones acerca de una fenomenología de la memoria incluyen dos formas fundamentales de recuerdo: la de la memoria individual y la de la memoria colectiva. En especial cuando se habla de memoria cultural, surge la pregunta acerca de quién es en realidad el sujeto de las operaciones de memoria. ¿De qué sujeto emanan estas operaciones? El interrogante acerca de si la memoria debe ser considerada como originariamente individual o colectiva se topa, de acuerdo con Ricœur, con un dilema, ya que, por un lado, no se sabe qué significa la afección en el presente viviente por un recuerdo de una imagen de una cosa pasada. Y por otro, no queda claro cómo se produce la búsqueda de un recuerdo perdido o reencontrado, y a qué sujeto deben atribuírseles tal afección y búsqueda (Ricœur, 2004, p. 125). En la concepción de Ricœur de la memoria cultural es fundamental la existencia de un lazo afectivo de las funciones del recuerdo con otros allegados o con extraños, así como con su atribución a sí.

En referencia a mis reflexiones sobre una fenomenología de la memoria, quiero señalar que la tensión que resulta de la confrontación de los conceptos de memoria individual y memoria colectiva debe ser resuelta en el terreno fronterizo entre fenomenología y ciencias sociales. Para la perspectiva fenomenológica son fundamentales en este sentido las reflexiones de Edmund Husserl sobre la conciencia interna del tiempo, las de Alfred Schütz sobre el conocimiento y la relevancia y las de Paul Ricœur sobre los sujetos de recuerdo. El foco de las ciencias sociales parte de la concepción de Maurice Halbwachs sobre la memoria colectiva, refiere a la relación dialéctica entre individuo y sociedad en los términos de Berger y Luckmann, y se sustenta en la teoría de Jan Assmann sobre la memoria cultural.

\section{La memoria cultural: la perspectiva de las ciencias sociales}

De acuerdo a Assmann, en la interrelación del "recuerdo" (vínculo con el pasado), la "identidad" (imaginación política) y la "continuidad cultural” (formación de la tradición), toda cultura desarrolla su "estructura conectiva (konnektive struktur)", la cual opera de forma unificante y vinculante en dos dimensiones: la social y la temporal (Assmann 2007, p. 16). El individuo está ligado a sus prójimos de tal modo que la cultura, en tanto "universo simbólico" (Berger y Luckmann, 1968, p. 131), 
abre un espacio de experiencia, expectativa y acción común que expresa una fuerza vinculante y funda confianza y orientación. El universo simbólico genera pertenencia e identidad, tanto a través de su aspecto normativo como de su aspecto narrativo, $y$ se basa en el saber común y en una autopercepción compartida (Assmann, 2007, p. 16). Peter L. Berger y Thomas Luckmann, que acuñaron el concepto de "universo simbólico", comprenden a la sociedad como realidad objetiva y subjetiva, poniendo siempre el foco en la relación dialéctica entre individuo y sociedad. Los autores parten del "individualismo metodológico" de Max Weber y sostienen que el pensamiento sociológico debe incluir siempre la perspectiva del actor individual. Con relación al efecto de los universos simbólicos, resulta fundamental que los mismos justifican y legitiman tanto la vida individual como el orden social-institucional. El universo simbólico trae orden a la actitud subjetiva hacia la experiencia personal. Experiencias de diferentes esferas de la realidad se integran a partir de un universo simbólico abarcador que, en general, puede ser entendido como cultura (Berger y Luckmann, 1968, p. 125).

La historia, en especial, es sistematizada a través del universo simbólico, en tanto todos los acontecimientos generales son colocados en una unidad interconectada a la que pertenecen el pasado, el presente y el futuro. Para el pasado se pone a disposición el "recuerdo" en el que todos los que pertenecen a una determinada sociedad pueden participar. Con relación al futuro, se garantiza un sistema de referencia común, un marco de proyección para las acciones individuales. El universo simbólico vincula a los hombres con sus predecesores y sucesores, y los coloca en un nexo de sentido. Es creada una totalidad significativa, que trasciende la finitud de la existencia individual y le brinda un sentido a la muerte del individuo. De esa manera los miembros de una sociedad pueden sentir que pertenecen a un mundo significativo. A través del universo simbólico, se genera un mundo significativo que estaba ahí antes de nuestro nacimiento y que estará ahí luego de nuestra muerte (Berger y Luckmann, 1968, p. 131).

Partiendo del concepto de Assmann de "estructura conectiva", que vincula entre sí a las dimensiones social y temporal de una cultura, se argumenta que las sociedades imaginan autopercepciones y que continúan una identidad a través de las generaciones formando una cultura del recuerdo. El modo en que tiene lugar el recuerdo puede ser diferente en diversas sociedades. En cada caso siempre debe investigarse "cómo recuerdan las sociedades y cómo [...] imaginan en tanto recuerdan" (Assmann, 2007, p. 18). Con el concepto de "memoria cultural", Assmann se refiere a una de 
las dimensiones exteriores de la memoria humana. En general tiende a pensarse a la memoria como un fenómeno puramente interior del que se ocupan la fisiología cerebral, la neurología y la psicología, y no las ciencias históricas, culturales y sociales. La capacidad y la dirección de la memoria cultural son, sin embargo, dependientes no de condiciones internas sino de condiciones externas, esto es, sociales y culturales (Assmann, 2007, p. 19).

Para Assmann y también para Ricœur - como veremos más adelante-, esta dimensión externa de la memoria, relevante en el sentido de una perspectiva sociológica, juega un rol central vinculado a las reflexiones de Maurice Halbwachs. Assmann diferencia cuatro dimensiones externas de la memoria: 1) la memoria mimética (rutinas de acción cotidianas que nunca están totalmente codificadas); 2) la memoria de las cosas; 3) la memoria comunicativa, en el sentido del lenguaje y la comunicación y; 4) la memoria cultural. También, en relación con la constitución de la conciencia individual, siempre se parte de factores de condicionamiento social. De acuerdo con Assmann, la conciencia y la memoria no son posibles de ser comprendidas psicológicamente; exigen una explicación "sistémica" en la cual esté envuelta la interacción con otros individuos. La conciencia y la memoria surgen consecuentemente a través de la participación en la interacción social (Assmann 2007, p. 20). Lo específico de la memoria cultural —en contraposición a los otros tres tipos de memoria- es para Assmann la transmisión de sentido a través de ritos y símbolos, entre otros medios. Las tres dimensiones externas recién mencionadas son efectivas en la memoria cultural cuando traspasan sus dimensiones de finalidad y refirieren, adicionalmente, a otra cosa que es fundadora de sentido. Así, las acciones rituales constituyen una forma de transmisión y de presentificación, y las cosas materiales pueden también cumplir esta función. Se considera que el pasaje de los mencionados tipos de memoria a la memoria cultural tiene lugar "más o menos sin solución de continuidad". El interés de Assmann (2007) consiste en explorar el significado del lenguaje y la comunicación para la memoria cultural.

La subsecuente argumentación de Assmann se sustenta en la observación de que la alfabetización de la comunicación permite una "expansión de la situación de comunicación más allá del almacenamiento temporario externo". Este proceso se institucionaliza y emerge en la forma de representaciones simbólicas en tres campos: la economía, la política y los mitos que aseguran la identidad. Aquí tienen lugar procesos de recuerdo y de olvido análogos a los de la conciencia individual. En la 
comunicación elementos de sentido son evacuados en el reservorio temporario y externo de la memoria cultural. Esto sucede por diferentes medios: a través del olvido colectivo, la represión o la censura. Es fundamental señalar que la memoria cultural no se limita a alimentar la tradición y la comunicación, sino que de forma análoga a la conciencia individual, va más allá de esto al permitir irrupciones de sentido "desde más allá del sentido en cada caso actualizado, recursos a lo olvidado, repristinaciones de la tradición, retorno de lo reprimido". Respecto a la especificación de la memoria cultural y a su analogía con el recuerdo de la conciencia individual, señala Assmann (2007): "Esta memoria es cultural porque solo puede ser realizada institucional y artificialmente, y es memoria porque funciona respecto a la comunicación social del mismo modo que la memoria individual respecto a la conciencia" (p. 23).

En cuanto a la distinción fenomenológicamente relevante entre memoria colectiva y memoria individual, la diferenciación de Assmann entre la cultura del recuerdo (erinnerungskultur) y la capacidad individual de recuerdo aparece como central. La cultura del recuerdo está caracterizada por una relación normativa con el pasado en la que se plantea la pregunta acerca de qué debemos recordar. Esta relación normativa con el pasado sería el presupuesto fundamental para la "formación de horizontes sociales de sentido y tiempo". Solo a través del recordar se crearía el pasado; a la relación con el pasado construida temporalmente estarían siempre vinculados el deseo, el pensamiento y la acción colectivas. La relación con el pasado se basaría en la percepción de discontinuidades, de rupturas y de renacimientos ligadas a ellas, representando la muerte la ruptura más grande (Assmann, 2007, p. 30). En el caso de la muerte, se pone de manifiesto de forma especialmente clara la diferencia entre el recordar individual y el recordar colectivo. Existe una diferencia entre el recordar del individuo que contempla retrospectivamente su vida desde su vejez, y el modo en que es conmemorada dicha vida por el mundo sucesor (nachwelt). En la conmemoración de los muertos se vuelve reconocible el elemento específicamente cultural del recuerdo colectivo. El muerto "sigue viviendo", por decirlo de algún modo, en el recuerdo del mundo sucesor, y pareciera que sigue viviendo por su propia fuerza. Es, sin embargo, la comunidad la que se decide voluntariamente a mantener en el presente el recuerdo del miembro fallecido. A diferencia de la tradición, que se basa en continuidades, para Assmann (2007) el recuerdo y el olvido sirven para el trato con discontinuidades y rupturas. 
La concepción de Halbwachs permite aquí una continuación de la argumentación: los colectivos, según esta posición, no pueden recordar, pero determinan el recuerdo de los individuos (Assmann, 2011). El sujeto de la memoria y el recuerdo es siempre el hombre individual, siendo decisivo ahora que el recuerdo del individuo está organizado por "marcos". Es resaltado que la memoria individual de una determinada persona se construye en virtud de su participación en procesos comunicativos. Cuando los marcos de referencia de la realidad comunicada se modifican, la consecuencia es el olvido. Assmann (2007) resalta "que solo el sentir (empfinden) es individual, pero no el recuerdo" (p. 36).

Otras conceptualizaciones son relevantes para la caracterización de las formas del recuerdo colectivo: con relación a los principios de la construcción social de la memoria colectiva, con su "forma de funcionamiento bimodal", se distingue entre memoria comunicativa y memoria cultural. La memoria comunicativa se refiere al pasado reciente e incluye recuerdos que el hombre comparte con sus contemporáneos. El recuerdo comunicativo o "biográfico" se basa en las experiencias personales y sus condiciones, que son transmitidas en la interacción social directa. La memoria cultural resulta del "recuerdo fundante", que se manifiesta en objetivaciones fijas (rituales, mitos, danzas, etc.). De acuerdo con Assmann (2007):
A diferencia de la memoria comunicativa, la memoria cultural es un asunto de mnemotécnica institucionalizada, a través de la cual la historia se convierte en mito y, solo de esa manera, adquiere realidad en el sentido de fuerza normativa y formativa duradera (p. 52).

La polaridad entre recuerdo comunicativo y cultural es caracterizada como una "estructura de participación", la cual está determinada por el reemplazo de los legos por los expertos como portadores y mediadores del recuerdo. La memoria cultural transportaría contenidos de sentido extra-cotidianos, lo cual se expresaría en el "exoneramiento de la cotidianeidad de sus [...] portadores" (p. 54). A través de la participación en la memoria cultural, se crea un terreno de sentido separado tajantemente de la cotidianeidad que tiene lugar en el "tiempo del sueño", constituyéndose así una relación de la comunidad con cosmologías. La vida humana tendría lugar así en una "temporalidad dual (zweizeitigkeit)". "La memoria cultural amplía y completa al mundo cotidiano con las otras dimensiones de las negaciones y potencialidades, y subsana de ese modo los acortamientos que le acaecen a la existencia 
por la cotidianeidad" (p. 57). Para Assmann, los planos del tiempo sagrado y del tiempo cotidiano no existen uno separado del otro; antes bien, el tiempo sagrado actúa fundando el sentido del cotidiano (p. 58).

\section{Fenomenología de la memoria}

Paul Ricœur habla incluso de una relación de competencia entre una mirada fenomenológica y una mirada sociológica de la memoria. Sin embargo, ambas perspectivas son para él centrales si se quiere diferenciar entre memoria individual y colectiva. Así, reconoce en la emergencia de la problemática de la subjetividad, y de la cada vez más apremiante posición egológica, tanto una problematización como un movimiento de retirada de la conciencia hacia sí misma. Se desarrolla una "escuela de la interioridad" cuyos tres máximos representantes serían San Agustín, John Locke y, el más extremo entre ellos, Edmund Husserl. La radicalización subjetivista que surge de esta escuela trae consigo la dificultad de que la atribución a un sujeto colectivo se vuelve, o bien impensable, o bien solo derivada y metafórica. En las ciencias humanas, especialmente en la sociología, se desarrolla una posición que le opone al individualismo metodológico un holismo metodológico, el cual fue representado sobre todo por la escuela durkheimiana y, más tardíamente, también por Maurice Halbwachs. Esta perspectiva sociológica concibe, a comienzos del siglo XX, a la "conciencia colectiva" como una de esas realidades a las que les corresponde un estatus ontológico que no puede ser puesto en cuestión. Para esta corriente de pensamiento antagónica a la escuela de la interioridad, concebir a la memoria individual como supuesta instancia originaria es una posición problemática que debe achacársele al psicologismo (Ricœur, 2004, p. 126).

En este campo de tensión entre individualismo metodológico y holismo metodológico, Ricœur encuentra una situación polémica en la cual una vieja tradición de la reflexividad se enfrenta con una tradición más joven de la objetividad, entrando también la memoria individual y la memoria colectiva en rivalidad. Ambas posiciones no se opondrían, sin embargo, en el mismo plano, sino que se trataría de mundos discursivos que se habrían vuelto extraños entre sí. El programa de Ricœur consiste en aclarar el malentendido radical entre estas posiciones y en tender un puente entre las mismas. En este sentido formula la hipótesis de que la memoria colectiva y la individual se constituyen de forma distinta, pero recíproca y entrecruzadamente. Ricœur propone el concepto de atribución como concepto operativo que puede 
producir una conmensurabilidad entre ambas perspectivas. Aquí debe diferenciarse entre la atribución a sí de fenómenos mnemónicos y su atribución a otros, extraños o próximos (Ricœur, 2004, p. 127).

Ahora me concentro decididamente en la perspectiva fenomenológica de la investigación de la memoria, aunque siempre refiriendo a la ya presentada posición de las ciencias sociales. Las reflexiones de Edmund Husserl son decisivas para una fenomenología de la memoria individual, ya que entablan una vinculación convincente entre tres problemáticas: la interioridad, la memoria y el tiempo. Para una confrontación de la perspectiva fenomenológica con la posición sociológica, son relevantes, de acuerdo a Ricœur, sobre todo las Lecciones sobre la fenomenología de la conciencia interna del tiempo (Husserl, 1928) y la quinta Meditación cartesiana de Husserl (1931), en la que se hace foco en el problema de la transición desde una egología hacia la intersubjetividad. En las Lecciones, se prepara el dominio de la egología, mientras que en la quinta Meditación cartesiana se aspira a una salida heroica en dirección a "comunidades intersubjetivas superiores" (Ricœur, 2004, p. 144). Ricœur se interesa por el esbozo de una fenomenología de la conciencia interna que se muestra adecuada para una contraposición de la rememoración (remémoration) y la conmemoración pública (commémoration) (p. 146). Respecto a la relación de la memoria individual y la colectiva, en una toma de distancia de una tematización de la conciencia interna del tiempo, en el punto de inflexión entre la teoría de la conciencia trascendental y la teoría de la intersubjetividad, Husserl intenta pasar del ego individual al otro, que a su vez puede constituir un nosotros. Dado que en la quinta Meditación cartesiana husserliana se habla de una "comunitarización (vergemeinschaftung) de la naturaleza física" (párr. 55), de una constitución de "comunidades intersubjetivas superiores" que surge de un proceso de "comunitarización social" (párr. 58), puede hablarse, en los términos de Ricœur, de una "memoria comunitaria" en conexión con mundos de la vida concretos, en los cuales las comunidades viven, actuando y sufriendo (p. 154).

Sin embargo, si la constitución del otro es concebida por Husserl desde una perspectiva egológica como partiendo de la esfera de propiedad (eigenheitssphäre), entonces este trascendentalismo cae en paradojas. Por un lado, se constituye al otro como extraño, es decir, como no-yo; y por otro lado, el otro es constituido "en mí", en tanto a través de la "apresentación (appräsentation)" se genera una analogía como parte de la comprensión del otro. En relación con la perspectiva de una sociología de la conciencia colectiva, puede remarcarse que esta última se desarrolla solo en un 
segundo proceso de objetivación de intercambios intersubjetivos. Debido al carácter análogo de las otras conciencias con la conciencia subjetiva, para Ricœur puede comprenderse a la memoria individual como análoga a la conciencia colectiva también en su forma de funcionamiento; el autor argumenta: “Gracias a esta traslación analógica, estamos autorizados a emplear la primera persona en la forma plural y a atribuir a un nosotros - cualquiera que sea su titular - todas las prerrogativas de la memoria" (Ricœur, 2004, p. 156). La representación de la memoria colectiva formada en un razonamiento por analogía considera a esta como una colección de huellas dejadas por acontecimientos que influenciaron el transcurso de la historia del grupo en cuestión, y que fueron celebradas como recuerdos comunes en fiestas, rituales y festejos públicos. Partiendo de esta transferencia analógica, Ricœur se ve habilitado a considerar a las "comunidades intersubjetivas superiores" como sujetos de inherencia de sus recuerdos, y a hablar de su temporalidad e historicidad. El ser en cada caso mío (jemeinigkeit) de los recuerdos podría, de forma análoga, ser ampliado a la idea de que somos propietarios de nuestros recuerdos colectivos, lo cual alcanzaría para anclar la historia escrita en la existencia fenomenológica de grupos. De este modo, podría hablarse también de una historia de las "mentalidades" o de las "culturas" (p. 156).

Cuando, partiendo de las reflexiones en el campo de tensión entre fenomenología y sociología, se pasa a investigar la relación entre recordar, memorizar y conmemorar, el problema de la atribución se muestra como fundamental. La memoria debe ser entendida, a la vez, como un caso particular y como un caso singular; un caso particular, puesto que los fenómenos mnemónicos son fenómenos psíquicos. Por tanto pueden ser atribuidos a cualquiera; su significado puede ser captado más allá de toda atribución explícita (Ricœur, 2004, p. 164). Respecto a la atribución, debe diferenciarse entre el "cumplimiento" del significado "que uno se atribuye a sí mismo" y la "apresentación" del significado "que se le atribuye a otro" (p. 166). Por último, Ricœur aboga por un acercamiento entre una fenomenología y una sociología de la memoria. Para ello, la primera debe dejarse influir por la segunda a los fines de convertirse en una fenomenología aplicada inmediatamente a la realidad social. Esta debe ocuparse de la participación de sujetos que están habilitados a comprenderse a sí mismos en diferentes niveles de reflexión consciente como autores de acciones. Con relación al ejercicio y la práctica de la memoria, pueden constatarse características que, para Ricœur, portan la marca del otro. Sobre todo en lo que respecta al lenguaje, se vuelve claro que el recuerdo expresado es un tipo de discurso que el sujeto se dice a sí mismo (p. 167). 
En relación con su ampliación de la fenomenología a la esfera social, Ricœur se topa con la obra de Alfred Schütz (1932), que concibe a la experiencia del otro como un dato tan originario como la experiencia de sí mismo. Disponemos de una creencia práctica en la existencia del otro porque actuamos con él, referidos a él y sobre él, y porque somos afectados por su acción. La fenomenología aplicada al mundo social se centra en el ordenamiento de la vida común humana, y los sujetos actuantes y sufrientes son concebidos, desde el vamos, como miembros de una comunidad o un colectivo. Una fenomenología de la realidad social está directamente relacionada con una fenomenología de la memoria, estando el fenómeno "transgeneracional", esto es, el fenómeno de la seguidilla de generaciones, anclado en el terreno de la comunidad. En términos de Schütz, se tematiza a los contemporáneos (zeitgenossen), los predecesores (vorgänger) y los sucesores (nachfolger), a partir de los cuales se forman, respectivamente, el "mundo de los contemporáneos (mitwelt)", el "mundo de los predecesores (vorwelt)" y "el mundo de los sucesores (folgewelt)". En este contexto, el mundo de los contemporáneos funciona como una "bisagra" que pone de manifiesto la "simultaneidad o cuasi-simultaneidad de la conciencia ajena con la propia" (Schütz, p. 170). Además, debe mencionarse el fenómeno de "envejecer juntos (gemeinsam altern)", que produce una sinergía entre dos duraciones en desarrollo. Esta perspectiva fenomenológica se centra en las interacciones y la configuración interactiva de identidades, tomando así distancia de una fenomenología que está "demasiado marcada con los fenómenos perceptivos y, en general, cognitivos” (Ricœur, 2004, p. 170).

Para concluir con las reflexiones de Ricœur sobre una fenomenología de la memoria, se define un plano intermedio de referencia que media entre los polos de la memoria individual y la memoria colectiva. Se trata del plano en el que tienen lugar los intercambios concretos entre la memoria viviente de los individuos y la memoria pública de las comunidades. Ricœur se concentra aquí en el plano de los "que están cerca, al que pertenecen aquellos hombres" que cuentan para nosotros y para los que nosotros contamos y que se perfilan en diferentes distancias entre el sí mismo y el otro. En un juego de distanciamiento y de acercamiento, se trata de acercarse y de sentirse cerca. En los términos de la antigüedad, la cercanía constituiría una respuesta de la amistad, la philia, que debe establecerse entre el individuo y el ciudadano como aquel que realiza un aporte a la politeia, a la vida y la acción de la polis. Los allegados se encuentran, simultáneamente, a medio camino entre el sí y el se, el uno (man), en el sentido de las relaciones de contemporaneidad señaladas por Alfred Schütz. Como remarca Ricœur (2004), los allegados son “otros próximos, otros privilegiados" 
(p. 171). Respecto a la atribución de memoria, este lazo que nos une con los allegados es fundamental. En él juegan un rol importante las relaciones de filiación y de pareja, como así también las relaciones sociales en las diferentes formas de la pertenencia y en diferentes órdenes de grandeza. Estas formas de relación y de grupos de pertenencia influyen en la contemporaneidad del "envejecer juntos", en el que los "acontecimientos" que limitan la vida humana - nacimiento y muerte - son de decisivo significado existencial. Los allegados son, además, aquellos que me apoyan en mi existencia y que, en el caso ideal, yo apoyo en apreciación igual y recíproca en la suya. La aprobación recíproca de los allegados se pone de manifiesto en el hecho de que cada uno puede vivir tanto sus poderes como sus incapacidades. En términos de Ricœur, yo espero de mis allegados que aprueben y den por bueno lo que atestiguo a través del habla, la acción y el relato, y que pueda atribuirme la responsabilidad por mis acciones. Entre mis allegados se cuentan también aquellos que desaprueban mis acciones pero no mi existencia (Ricoeur, 2004). Como síntesis de sus reflexiones sobre una fenomenología de la memoria, Ricœur presenta no solo una polaridad entre la memoria individual y la colectiva, sino que también esboza una triple atribución de la memoria: al sí mismo, a mis allegados y a los otros.

\section{Memoria y relevancia}

Ahora completaré las reflexiones fenomenológicas y de las ciencias sociales acerca del interjuego entre la memoria individual y la memoria colectiva a través de las teorías schützianas del mundo de la vida (lebenswelt) y de la relevancia, con el objetivo de resaltar el significado de la perspectiva egológica para la perspectiva colectivista. Aquí es central el concepto de "mundo de la vida”, que Schütz retoma de Husserl de forma modificada. Como parte privilegiada de nuestro mundo de la vida subjetivamente fijado, Schütz define al mundo cotidiano, como la realidad que el adulto en "actitud natural" encuentra como simplemente dado. La "actitud natural" es el estado de conciencia en el cual el hombre toma esta realidad cotidiana como dada. Ella es dominada por el motivo pragmático, lo cual significa que el mundo cotidiano se nos manifiesta como un mundo que podemos influir y cambiar a través de nuestro efectuar y actuar (Schütz, 1945, p. 208). Al mundo de la vida pertenece tanto la capa de sentido cultural, que convierte objetos físicos en objetos de la experiencia ingenua, como también el mundo social. Sin embargo, tal como Schütz utiliza el concepto, el mundo de la vida abarca más que la realidad cotidiana. El hombre se hunde en estado de sueño y cae en mundos fictivos a través de fantasear, abandonando así la actitud 
natural. Además, está capacitado a trascender el mundo cotidiano mediante símbolos y puede, como excepción, incluso conscientemente pasar de la actitud natural a otro terreno de realidad. Los terrenos de realidad extracotidianos que podemos compartir con otros hombres son la religión, la política, la ciencia, el arte, etc. Con ayuda de símbolos podemos comunicarnos respecto a estas realidades. Para la problemática de la memoria se muestra como fundamental el hecho de que debe partirse de una "historicidad del mundo de la vida" (Luckmann, 1991, p. 195) enraizada como momento fundamental de la experiencia en capas elementales de la temporalidad de la experiencia del lebenswelt. La localización del hombre individual en el mundo de la vida es histórica, ya que está determinada por un a priori sociohistórico, en el que el hombre se vuelve consciente de sí mismo de forma autoevidente y preteórica (Dreher, 2007, p. 18).

$\mathrm{Si}$-siguiendo a Schütz- nos concentramos en el análisis de la memoria individual, debe señalarse que la conciencia subjetiva del individuo es fundamental para el mundo social y viceversa, ya que la primera está siempre inmersa en el último. Lo social no es algo que se adose necesariamente a las estructuras de tipificación existentes cuando las mismas son realizadas concretamente, sino que es esencial para todo patrón de tipificación (Barber, 2001, p. 104). Esto significa que nuestra percepción, nuestro pensamiento y nuestro recuerdo en el mundo de la vida está predefinido por la socialidad a través del conocimiento que es transmitido al individuo mediante la socialización. De modo similar a lo que sucede en Assmann, aquí se considera que la conciencia y la memoria deben explicarse a partir de contextos de interacción. Según Schütz (2003):

El hombre experimenta el mundo social en que ha nacido, y dentro del cual debe orientarse, como una apretada trama de relaciones sociales, de sistemas de signos y símbolos, con su particular estructura de sentido, de formas institucionalizadas, de organización social, de sistemas de estatus y prestigio, etc. Todos los que viven dentro del mundo social presuponen el sentido de todos estos elementos en toda su diversidad y estratificación, así como el esquema de su trama (p. 214).

Los hombres socializados vivencian su cotidianeidad en el modo de la tipicidad. Las diferentes cosas que los hombres perciben no son experimentadas meramente como una seguidilla de fenómenos nuevos y únicos, sino más bien como tipos. La 
mayor parte del saber del individuo tiene origen social y fue transmitido a él como herencia por padres y maestros. Esta herencia consiste en un conjunto de sistemas de tipificaciones relevantes, de soluciones típicas para problemas prácticos y teóricos y de instrucciones de comportamiento típico (Schütz, 1955, p. 208). Para una fenomenología de la memoria, es central que estos sistemas de tipificaciones tienen la función del ordenamiento y de la presentificación de representaciones vinculadas al pasado y que pueden, en este sentido, ser entendidos como marco de la memoria. Respecto al conocimiento recordado, fijan también sistemas de relevancia que estructuran el conocimiento tipificado. Las típicas y los sistemas de relevancia pueden ser vistos como fundamento de la realidad en cada caso válida y aprobada, y del pasado en cada caso válido. Así vistas, son fundantes para la memoria individual y social (Sebald, 2012, p. 203).

Las reflexiones de Schütz acerca de una teoría del símbolo pueden ponerse fructuosamente en vinculación con las de Assmann. A través de la utilización de símbolos, estamos capacitados a experimentar y comunicar ideas extracotidianas, ideas y representaciones "trascendentas a la cotidianeidad" que, por ejemplo, pertenecen al terreno de la política o de la religión. Los símbolos mismos — por ejemplo, los artefactos de los que se sirve el recuerdo- son elementos del mundo cotidiano, del mundo de eficacia (wirkwelt), en el que podemos comunicarnos. Son portadores de significados trascendentes a la cotidianeidad insertos en la comunicación. Los símbolos del pasado que ponen a disposición nexos de sentido en el presente son, como vimos en Assmann, las instancias esenciales de mediación de la memoria cultural.

Desde mi perspectiva, la teoría de la relevancia de Schütz es especialmente adecuada para tender un puente entre una perspectiva científico-social de la memoria colectiva y una perspectiva fenomenológica de la memoria individual. El concepto schütziano de relevancia es utilizado como principio regulativo para la mediación entre la experiencia subjetiva y el saber objetivado, poniendo el foco en la estructura del saber del mundo de la vida, que es experimentado en diferentes planos de realidad. El conocimiento que tenemos del mundo, tanto en el pensamiento cotidiano como en el científico, consiste en constructos, esto es, en abstracciones, generalizaciones, formalizaciones e idealizaciones que son específicas para una determinada organización del pensamiento. En este contexto, es fundamental para el científico social, a diferencia del científico natural, que los hechos, acontecimientos y datos que atañen al mundo social no existen de forma pura. El mundo social tiene una estructura de sentido y 
relevancia especial para los hombres que piensan y actúan en él. En este sentido, el conocimiento sobre el mundo social está siempre ya preinterpretado (Schütz , 1953, p. 5). Esta estructuración especial de nuestro mundo social se sustenta en tipificaciones y simbolizaciones que están predeterminadas como dadas indudablemente; las mismas son dependientes de los esquemas de expresión e interpretación socialmente condicionados del grupo al que pertenecemos, que en general es denominada la "cultura" de nuestro grupo . El saber del individuo se articula en diferentes grados de familiaridad. Qué conocimiento es tomado por el individuo como familiar, creido o desconocido y cuál no, es relativo. En esta escala de relevancia, los allegados de los que habla Ricœur se encuentran entre lo familiar y lo extraño.

Si nos concentramos en el conocimiento e investigamos la experiencia de su estructura individual, encontramos que los correlatos subjetivos de este saber dependen de la motivación individual. El individuo al que se le revela el mundo se experimenta a sí mismo siempre en una determinada situación que debe definir. Esta situación está marcada por dos momentos diferentes: un componente de la definición de la situación resulta de la estructura ontológica del mundo predado, mientras que la otra remite a la situación biográfica del individuo, que incluye un acervo de conocimiento específico. Los componentes ontológicos de la situación del individuo son sentidos como impuestos - algo le pasa al individuo sin la posibilidad de reaccionar espontáneamente a la situación predada. La situación biográfica, sin embargo, determina el carácter espontáneo de la situación dentro del marco ontológico (Schütz, 1957, p. 323). Con estas reflexiones, por un lado, se puede mostrar en qué medida el individuo es determinado por la estructura del mundo y por reservorios de conocimiento impuestos que influyen en su experiencia y su acción. Y por otro, puede explicar cómo partiendo de allí, el individuo está habilitado a actuar espontáneamente de acuerdo con determinados intereses. El concepto de relevancia de Schütz es, de acuerdo a Hisashi Nasu (2008), "el más importante principio regulativo de la construcción de la realidad, ya que coordina el saber sobre objetos con su experiencia y le sirve al actor individual para definir su situación" (pp. 91-93). Con la noción de relevancia, puede mostrarse en qué medida el individuo dispone de constelaciones de intereses específicos en una determinada situación biográfica, constelaciones que son responsables de las experiencias y las decisiones de acción.

Desde una perspectiva fenomenológica, para el análisis de la memoria, cuyo portador es en Schütz siempre el actor individual, debe ponerse el foco sobre todo en la 
diferenciación de dos sistemas de relevancia, a saber: las relevancias "intrínsecas" y las relevancias "impuestas". En ambos sistemas se trata de construcciones ideal-típicas que solo raramente aparecen en forma pura en la vida cotidiana. Las "relevancias intrínsecas" son el resultado de nuestros intereses elegidos; los mismos fueron establecidos por nuestra decisión espontánea, por el pensamiento que resuelve problemas o por acciones orientadas a un objetivo. En principio somos libres para elegir, pero este interés determina, una vez que existe, el sistema de los intereses esenciales elegidos o de relevancias intrínsecas. El interés del que surgen las relevancias intrínsecas es erigido por nuestra elección espontánea, sin embargo, podemos en cada momento desplazarnos más allá del foco del interés y modificar las relevancias intrínsecas (Schütz, 1946, p. 128). Las relevancias intrínsecas están conectas sobre todo con aquello que Assmann describe como memoria comunicativa, puesto que la misma resulta de interacciones directas con contemporáneos que pueden ser influenciados individualmente. El recuerdo biográfico es el de un individuo único con un pasado personal específico que se ha formado por las experiencias personales, pudiendo depender también las condiciones básicas de la experiencia y de la interacción con otros de relevancias impuestas.

Como argumenta Schütz (1946), no somos solo "centros de espontaneidad que se insertan en el mundo y provocan cambios en él, sino también meros receptores pasivos de eventos más allá de nuestro control que ocurren sin nuestra interferencia" (p. 127). Para nosotros son relevantes situaciones y acontecimientos que no son compatibles con nuestros intereses libremente elegidos, que no surgen de nuestras libres acciones y que debemos aceptar como son, sin tener la posibilidad de cambiarlos espontáneamente. Estas relevancias impuestas son oscuras y en general incomprensibles, y no pueden vincularse sin más con las relevancias intrínsecas; no disponemos del poder de cambiarlas a través de nuestras actividades. Las relevancias impuestas influyen solo parcialmente en la memoria colectiva y estructuran en cierto sentido también las experiencias biográfica y comunicativa. Es también la memoria cultural la que pone a disposición las realidades extracotidianas importantes para el individuo - representaciones religiosas, ideas políticas, etc. - y que se las impone al individuo como relevancias. El universo simbólico puesto de manifiesto por la memoria cultural influye entonces en las relevancias individuales, ya que de él parte continuamente una fuerza normativa y formativa. En conexión con la diferenciación de memoria comunicativa y colectiva, podría también hablarse de la distinción entre relevancias motivadas cotidiana y extracotidianamente. En el interjuego entre 
relevancias intrínsecas e impuestas, se fijan interpretaciones del mundo ya listas que pueden estar determinadas biográfica, cultural y socialmente. El mundo de la vida del individuo acepta, a la vez, interpretaciones mágicas o religiosas y explicaciones científico-naturales. En este contexto, es fundamental que la participación en la memoria comunicativa y en la memoria cultural dejan abiertas para el individuo ciertas libertades de elección motivadas intrínsecamente.

\section{Conclusiones: el pathos del holocausto y la memoria individual}

Presentaré ahora mis consideraciones finales acerca de la relación antagónica entre la memoria individual y la colectiva, valiéndome del ejemplo del recuerdo del holocausto. En este caso, también surgen los interrogantes acerca de a qué sujetos se les atribuye el recuerdo y, especialmente, de cómo los puntos de vista emocionales, que fueron mencionados por Ricœur con relación a los allegados, determinan el recuerdo. Para el análisis del recuerdo del holocausto Aleida Assmann utiliza el concepto de pathos, con el cual se introducen aspectos emocionales en el discurso de las ciencias culturales, aspectos que habían sido dejados de lado por los abordajes constructivistas y performativos. El concepto de pathos es tomado de la estética aristotélica del efecto y de la retórica y declarado como una energía que afecta al espectador, al observador y al lector no solo de modo momentáneo y puntual. El pathos es además responsable de que determinadas experiencias e historias sean más inolvidables que otras que no pueden imponerse como figuras de recuerdo sobreviviendo a los cambios de épocas y generaciones (Assmann, 2011, p. 10).

El funcionamiento conjunto de la memoria individual y la colectiva en lo que respecta al recuerdo del holocausto se vuelve especialmente tangible a través de la relevancia del testimonio. Testigos individuales sobrevivientes del holocausto y también testigos secundarios, esto es, terceros no participantes que documentan el sufrimiento de otros, son consultados como instancias fundamentales para el recuerdo del holocausto. Se plantea la pregunta acerca de cómo el sufrimiento experimentado individualmente pasó a los testigos de la época en la memoria cultural. Ocupándose del pathos del holocausto, Assmann reconoce un nuevo tipo de testigo relacionado con el proceso de superación de las explosiones de violencia del siglo XX, a saber: el "testigo moral". Actualmente, los relatos orales de sufrimiento de sobrevivientes del holocausto pasan a la memoria cultural de largo plazo. Aquí juega un rol importante la posibilidad de 
recurrir a videos de relatos de experiencias de sobrevivientes. Los relatos de experiencia constituyen para Assmann "el fundamento de un ethos secular y universal que, bajo el signo de los derechos humanos, dignifica la historia del sufrimiento del individuo" (Assmann, 2011, p. 19). Así se vuelve reconocible una figura del recuerdo que no genera ningún lazo grupal particular y afectivo; los individuos de los relatos de experiencias individuales de los testigos coexisten yuxtapuestos y no producen una narrativa unificada.

La particularidad del testimonio moral consiste en que le transmite una "percepción sensorial inmediata" a terceros en tanto documenta, y de ese modo objetiva, las atrocidades del nacionalsocialismo vivenciadas por él. A través de la publicación de una oral history se procuró que las dimensiones de la experiencia sean asimiladas en la escritura de la historia. Al testimonio del testigo moral le corresponde un contenido especial de verdad en el sentido de una "verdad corporalizada", puesto que en él se unen la víctima y el testigo. La violencia se inscribió en este caso en los cuerpos y en las almas de las víctimas/testigos. La verdad de su existencia corporal es más importante que el contenido de sus dichos. Según Assmann (2011), los testigos morales construyen una comunidad moral traspasando los límites institucionales. Apelan a ella pero no son "especialistas en la verdad revelada. Lo que tienen para ofrecer es una construcción subjetiva de la situación extrema por la que tuvieron que pasar" (p. 28). A través de la objetivación del recuerdo subjetivo en el marco del testimonio, se logra que el recuerdo individual se transforme en colectivo. El testigo avala una verdad que ha vivenciado en el propio cuerpo. La documentación y difusión mediática de esta perspectiva subjetiva genera que su constitución de verdad pase a la memoria colectiva, o para ser más precisos, a la memoria cultural. Su testimonio puede, en términos de Husserl, ser entendido como reservorio de conocimiento de una "comunidad intersubjetiva superior", ya que el surgimiento de este contenido de memoria está desde el "vamos" inmerso en contextos sociales.

El recuerdo individual del testigo moral resulta de los sistemas de relevancia impuestos e intrínsecos de la situación biográfica específica de la experiencia del holocausto, y es además presentada en la actualidad a partir de sistemas de relevancia específicos que expresan la individualidad del testigo. En este sentido, a los testigos morales se les "atribuye" el recuerdo, cuya construcción subjetiva pasa a la memoria colectiva en el sentido del oral history. Siguiendo la argumentación de Ricœur, estos testigos son entendidos como aquellos otros a los que se les asigna una afección y que, a través del 
pathos, se les manifiesta una ligazón afectiva. De este modo, los testigos morales son comprendidos como portadores de la memoria. De ellos emana una comunitarización moral que traspasa los límites institucionales. Desde una perspectiva fenomenológica, puede mostrarse así cuáles son los presupuestos gnoseológicos que determinan el recuerdo individual y el recuerdo colectivo del holocausto. La perspectiva sociológica reconstruye la situación sociohistórica concreta que es documentada por los testigos - las atrocidades del nacionalsocialismo- que se inscriben en la memoria cultural de la sociedad.

\section{Referencias}

Assmann, A. (2011). Pathos und passion. Über gewalt, trauma und den begriff der zeugenschaft. In G. Hartmann \& A. Assmann (Eds.). Die Zukunft der Erinnerung und der Holocaust, (pp. 9-40). Konstanz: Konstanz University Press.

Assmann, J. (2007). Das kulturelle gedächtnis. Schrift, erinnerung und politische identität in frühen hochkulturen. München: Beck.

Barber, M. D. (2001). Equality and diversity: Phenomenological investigations of prejudice and discrimination. Amherst, NY: Humanity Books.

Berger, P. L., y Luckmann, T. (1968). La construcción social de la realidad. Buenos Aires: Amorrortu Editores.

Dreher, J. (2007). Lebenswelt, identität und gesellschaft-sozialtheoretische reflexionen zwischen phänomenolgie, wissenssoziologie und empirischer forschung. In T. Luckmann (Ed.). Lebenswelt, identität und gesellschaft. Schriften zur wissens-und protosoziologie, (pp. 7-23). Konstanz: UVK.

Harris, S. R. (2000). The social construction of equality in everyday life. Human studies, 23(4), 371-393.

Husserl, E. (1928). Vorlesungen zur Phänomenologie des inneren Zeitbewußtseins. Halle a. S. Niemeye.Husserl, E. (1931). Cartesianische meditationen. Eine einführung in die phänomenologie. In, G. Schriften \& E. Ströker (Eds.). Hamburg: Meiner. 
Luckmann, T. (1991). Geschichtlichkeit der lebenswelt? In J. Dreher (Ed.). Lebenswelt, identität und gesellschaft. Schriften zur wissens-und protosoziologie, (pp-193-205). Konstanz: UVK.

Nasu, H. (2008). A continuing dialogue with Alfred Schutz. Human studies, 31(2), 87-105.

Ricœur, P. (2004). La memoria, la historia, el olvido. Buenos Aires: Fondo de Cultura.

Schütz, A. (1932). Der sinnhafte aufbau der sozialen welt. Eine einleitung in die verstehende soziologie. In M. Endreß \& J. Renn (Eds.). Werkausgabe, Bd. II. Konstanz: UVK.

Schütz, A. (1957). Strukturen der lebenswelt. In M. Endreß \& I. Srubar (Eds.). Alfred Schütz Werkausgabe, Bd. V. 1. Theorie der lebenswelt 1. Die pragmatische schichtung der lebenswelt, (pp. 325-347). Konstanz: UVK.

Schütz, A. (2003). La igualdad y la estructura de sentido del mundo de la vida. Escritos II. Estudios en teoría social, (pp. 210-251). Buenos Aires: Amorrortu.

Schütz, A. (1946). The well-informed citizen. In A. Brodersen (Ed.). Collected papers II: Studies in social theory, (pp. 120-135). Den Haag: Martinus Nijhoff.

Schütz, A. (1955). Symbol, reality and society. In M. Natanson (Ed.). Collected papers I: The problem of social reality, (pp. 287-257). Den Haag: Martinus Nijhoff.

Schütz, A. (1945). On multiple realities. In M. Natanson (Ed.). Collected papers I: The problem of social reality, (pp. 207-260). Den Haag: Martinus Nijhoff.

Schütz, A. (1953). Common-sense and scientific interpretation of human action. In M. Natanson (Ed.). Collected papers I: The problem of social reality, (3-48). Den Haag: Martinus Nijhoff.

Sebald, G. (2012). Relevanzen und typiken. Zur phänomenologie von sozialen gedächtnissen. In J. Dreher (Ed.). Angewandte phänomenologie. Zum spannungsverhältnis von konstruktion und konstitution, (195-208). Wiesbaden: Springer VS. 